\title{
Orientation of particle attachment and local isotropy in diffusion limited aggregation (DLA)
}

\author{
Chi-Hang Lam \\ Department of Applied Physics, Hong Kong Polytechnic, Hung Hom, Hong Kong.
}

\begin{abstract}
We simulate 50 off-lattice DLA clusters, one million particles each. The probability distribution of the angle of attachment of arriving particles with respect to the local radial direction is obtained numerically. For increasing cluster size, $N$, the distribution crosses over extremely accurately to a cosine, whose amplitude decreases towards zero as a power-law in $N$. From this viewpoint, asymptotically large DLA clusters are locally isotropic. This contradicts previous conclusions drawn from density-density correlation measurements [P. Meakin, and T. Viscek, Phys. Rev. A 32, 685 (1985)]. We present an intuitive phenomenological model random process for our numerical findings.
\end{abstract}

PACS numbers: $61.43 \mathrm{Hv}, 05.40+\mathrm{j}$ 


\section{INTRODUCTION}

The diffusion limited aggregation (DLA) [1] is a fractal growth model exhibiting great complexity [2]. The properties of asymptotically large clusters are of fundamental importance. Some of these properties are independent of many details of the aggregation rules and are expected to be shared by large clusters grown in various experimental or natural situations. Efficient algorithms and improved computational facilities has enabled the generation of very large off-lattice clusters with more than 100 million particles [3]. However, many asymptotic properties of DLA are still unclear. There have been many debates on basic issues including the scaling behavior of large clusters and the multi-fractal properties of the growth probability measure [2].

This work concentrates on one of the most fundamental geometrical aspects, namely the local isotropy, of large off-lattice DLA. Meakin and Viscek « found that the two points density-density correlation is anisotropic. The tangential correlation decays algebraically with exponent $\alpha_{\perp} \simeq 0.41$, which is different from the radial exponent $\alpha_{\|} \simeq 0.29$. As a result, the geometry, in particular the orientation, of a segment of the cluster is related to its position inside the cluster. This behavior does not hold for some deterministic fractal curves containing spirals such as the Koch curve [5] in the limit of small segments. Asymptotically large DLA would be locally isotropic only if the two very different exponents converge to the same value. Such a trend was not identified [4] and the anisotropy has been assumed to persist asymptotically [4,11]. Here, we apply a substantially more accurate method and obtain the first unambiguously evidence of a systematic decrease in the local anisotropy of DLA for increasing cluster size. The trend of the decrease strongly indicates that asymptotically large clusters are locally isotropic, contradictory to previous conclusions [4].

Specifically, we investigate the orientation of particle attachment. For each new particle

in the cluster, let $\vec{R}^{\prime}$ and $\vec{R}$ be the position vectors relative to the center the cluster of respectively the new particle and its parent, where the parent is the particle in the aggregate upon which the new attachment is made. We define the center of the cluster to be at the seed. 
The vector $\vec{R}$ characterizes the local radial direction of this sticking event and $\vec{r}=\vec{R}^{\prime}-\vec{R}$ gives the sticking direction. The angle of attachment $\theta(-\pi<\theta \leq \pi)$ is defined as the

angle measured counter-clockwise from $\vec{R}$ to $\vec{r}$. Attachment with with $|\theta|<\pi / 2$ can be described as forward. We focus on the probability distribution $P(\theta, N)$ of $\theta$, which, in general, depends on the number of particles, $N$, in the cluster. If DLA were compact with nearly circular boundary, the above definition implies that $\theta$ is always close to zero and a backward attachment is geometrically impossible. The distribution $P(\theta, N)$ should peak at $\theta=0$. Although, DLA is far from being compact, at least for small clusters, forward attachment is favored for similar geometrical reason.

\section{SIMULATIONS AND RESULTS}

We compute the distribution $P(\theta, N)$ for $N=150 \times 2^{k}$, where $\mathrm{k}=0$ to 12 corresponding to $\mathrm{N}=150$ to 614400 . For each $k$, we histogram the values of the sticking angle $\theta$ for the $\left(100 \times 2^{k}+1\right)$-th to the $\left(200 \times 2^{k}\right)$-th particles. Proper normalization gives $P(\theta, N)$. The symmetry $P(\theta, N)=P(-\theta, N)$ is used. We averaged the results over 50 clusters. The statistical error is estimated from the sample to sample fluctuations.

Figure 1 shows $P(\theta, N)$ for 5 values of $N$. We used 16 bins to histogram. The error bars are smaller than the symbols except for $N=150$. The errors are as small as about $0.2 \%$ at $N=614400$, where our data is most accurate. For small $N$, forward sticking dominates so that $P(\theta, N)$ peaks at $\theta=0$ as expected. Backward stickings are rare. The peak broadens as $N$ increases. At $N=614400$, the ratio $P( \pm \pi, N) / P(0, N)$ between perfectly backward and forward attachments becomes about 0.31. Figure 1 show least square fits to:

$$
P(\theta, N)=1 / 2 \pi+a_{1}(N) \cos (\theta)
$$

for the 3 larger values of $N$, where $1 / 2 \pi$ ensures normalization. At $N=614400$, the quality of the fit amazing. There is apparently no systematic trend of deviation, as the data points scatter around the fitted curve by amounts comparable to the statistical errors. The fit is 
still good at $N=76800$, but at $N=9600$, there are noticeable systematic deviations. The quality of the fit is restored when we consider one more term in the cosine expansion:

$$
P(\theta, N)=\frac{1}{2 \pi}+\sum_{n=1}^{\infty} a_{n}(N) \cos (n \theta)
$$

Figure [ 1 shows the two parameters fits for $N=150$ and 1200. More generally, we fitted our complete data set of $P(\theta, N)$ with the first 4 cosine terms. Figure $2(a)$ plots the values of the amplitudes $a_{n}(N)$ in semi-log. We used histograms of 64 bins and the result agrees with those with 16 bins. We also computed $a_{n}(N)$ by Fourier transform and obtained again the same results. Figure 2(b) and (c) shows respectively $a_{1}(N)$ and $a_{2}(N)$ in $\log -\log$ plots. The linearity at $N \gtrsim 300$ implies:

$$
a_{n}(N) \simeq A_{n} N^{-\gamma_{n}}
$$

for $n=1$ and 2, where $\gamma_{1}=0.0997(3), A_{1}=0.309(2), \gamma_{2}=0.67(3)$ and $A_{2}=1.2(3)$. The bracketed values are the fitting errors. For $n \geq 3$, the measured $a_{n}(N)$ is not precise enough for a test of the above algebraic decay.

We also studied the closely related problem of the branch orientation of DLA. We adopt the Horton-Strahler's scheme of branch ordering [7]. The smallest branches without sidebranch are assigned order 1 and the main stems have the highest orders. Other authors have used slightly different schemes [8,9], which should give similar results to those reported here. From visual examinations of the branches shown in Ref. [8], it is evident that those with the highest order branches are nearly radial and the directedness decreases for lower orders. Quantitatively, we define the branch orientation angle $\phi$ to be measured counter-clockwise from the position vector of the base of the branch to the branch orientation vector pointing from the base to the tip. Figure 3 plots the probability distribution $P(\phi, s)$ of $\phi$ for the branch order $s=1$ to 5 . The data was averaged over 50 clusters, one million particles each. The distribution $P(\phi, s)$ can be approximated reasonably well by a cosine curve for $n$ close to 1 . In the cosine expansion of $P(\phi, s)$, the coefficient of the $\cos (\phi)$ term decreases rather quickly with $s$, while that of $\cos (2 \phi)$ is negative and rising up towards 0 . The coefficients as functions of $s$ are not well described by simple functional forms. 


\section{PHENOMENOLOGICAL MODEL}

We now propose an intuitive phenomenological picture for the properties of the angle of attachment. For convenience in presentation, we examine the following approach of DLA growth, instead of the more efficient random walker method. To add a particle to the cluster, the Laplacian potential is solved to obtain the particle probability flux lines. The new particle is launched randomly with uniform probability on a big circle inscribing and far away from the cluster. It subsequently traces the flux line deterministically towards the cluster until it hits and become part of it. In this scheme, the direction of the attachment is simply the tangent to the flux line at the point of contact. Figure 4 shows 200 flux lines with uniformly spaced starting positions far away. They are all equally likely to represent the next growth step. We will concentrate on the geometry of these typical flux lines which are relevant to the growth. Lines with very low probability weight, which can have very different geometry [10, are neglected.

The flux lines proceed nearly exactly radially inwards until they are close enough to the

cluster to be influenced by the geometry of the main branches. At this stage, the details of the side-branches are still unimportant. Depending on the position of the flux line relative to the nearest main branch, it can keep proceeding radially towards the tip or make a turn to approach the branch from either side. After advancing further, the geometry of the nearest side-branches becomes important. Again, the flux line approaches either towards the tip or one of the sides of the side-branch. Similar situation recurs for side-branches of the side-branches until the geometry of individual particles becomes relevant.

The typical total number of turns thus equals the number of levels of side-branching, which is proportional to $\tau=\ln N$ [8,9]. The angle of attachment is approximately the sum of the angles of turning. Suppose that when $\tau$ is increased by $\Delta \tau$, there are, on average, one more turn in the flux lines. We approximate the corresponding evolution of the distribution of the angle of attachment by:

$$
P(\theta, \tau+\Delta \tau)=\int_{-\pi}^{\pi} G\left(\theta-\theta^{\prime}, \Delta \tau\right) P\left(\theta^{\prime}, \tau\right) d \theta^{\prime}
$$


where there is a reparametrization with $\tau$. Implicit assumptions includes the uncorrelation of the turns and their isotropy so that the propagator $G$ only depends on $\theta-\theta^{\prime}$. Unfortunately, a direct numerical verification of Eq. (4) with the determination of $G$ requires computation of the flux lines for very large clusters, which is well beyond the capability of the relaxation method [2]. The validity of the assumptions can only be justified by comparing their predictions with simulations.

We consider first the simplest case where $G$ is a Gaussian centered at $\Delta \theta=0$ with width smaller than $\pi$. Equation (4) reduces to:

$$
\frac{\partial}{\partial \tau} P(\theta, \tau)=\nu \frac{\partial^{2}}{\partial \theta^{2}} P(\theta, \tau)
$$

where $\nu$ is the diffusion constant. The set of Eqs (2) and (3) is indeed a solution to Eq. (5). They predict $\gamma_{n}=\nu n^{2}$ and thus the amplitudes of the higher harmonics decay much faster. This is consistent with the practically zero values of the measured amplitudes for $n \geq 3$. Moreover, the result $\gamma_{2} / \gamma_{1}=4$ is in the same order as the numerical value $6.7(3)$. For general $G$, we expand:

$$
G(\Delta \theta)=\frac{1}{2 \pi}+\sum_{n=1}^{\infty} \frac{G_{n}}{\pi} \cos (n \Delta \theta)
$$

Substitution into Eqs (2) and (3) gives $\gamma_{n}=-\ln G_{n} / \Delta \tau$. Consider the next simplest form besides the Gaussian: $G(\Delta \theta) \sim \exp \left[-\left(\Delta \theta / \theta_{0}\right)^{4}\right]$. By matching $\gamma_{1}$ and $\gamma_{2}$ with the numerical values, we obtain respectively $\theta_{0} \equiv 94^{\circ}$ and $\Delta \tau=\ln 130$. It means that a flux line make a turn of typically not more than $94^{\circ}$ per increase in $N$ by a factor of 130 . For a cluster of $N=10^{6}$, each flux lines has roughly $N_{t} \simeq 2.8$ turns. We also tried $G$ in a very different form of the sum of two Gaussian peaks. It gives $N_{t} \simeq 4$.

Recall that the number of turns $N_{t}$ equals approximately the number of levels of sidebranching probed by the flux lines. The maximum branch order in a cluster of a million particles is typically 9 [9]. However, most flux lines terminate around the tips of the cluster so that the higher order branches, which reside close to the center, are irrelevant. As a result, $N_{t} \ll 9$. It seems that $N_{t} \simeq 3$ or 4 obtained from the very crude models are reasonable 
approximates. Even for large clusters, $N_{t}$ is very small. This is the cause of the extremely slow evolution of $P(\theta, N)$.

The length scale of the problem decreases as we consider deeper levels of side-branching. In our construction with the modified Gaussian, the displacement of the flux line in between turns typically decreases by a factor of $130^{1 / D} \simeq 17$ after every turn, where $D \simeq 1.72$ is the fractal dimension of DLA [2]. every flux line converges rapidly to its point of contact. For extremely large clusters and length scales in between that of the cluster and the individual particles, segments of flux lines containing their points of contact are statistically similar to each other after proper rescaling. This scaling property is a consequence of the approximation that the propagator $G$ is independent of $N$, based on the assumption that the lines progress in self-similar environments. Dependence of $G$ on $N$ should reveal in discrepancies from Eq. (3), which corresponds to any non-linearity in the log-log plot in Fig. 2(a) and (b). Within our accuracy, we cannot identify any unambiguous non-linearity for $N \gtrsim 300$. Our measurement is not sensitive to the well known deviations from self-similarity of DLA [2.:11].

Although the uncorrelation of the turns seems to be a reasonable approximation, the are obviously strong correlations within a turn so that Eq. (四) cannot be reformulated with smaller $\Delta \tau$ corresponding to fractions of a turn. Indeed, $G(\Delta \theta, \Delta \tau / 2)$ is not well defined in both models with the modified Gaussian and the double Gaussian, since $G_{3}<0$.

The behavior of the distribution $P(\phi, s)$ of the branch orientations can be accounted for similarly. The main branches with the highest orders are nearly radial. Their side-branches are attached to either sides with a subtended angle around $\pm 40^{\circ}$ [9]. This contributes to a step in the randomization of the orientation analogous to the turns of the flux lines. Therefore, much of above discussion applies. However, the correlation in the randomization process is stronger here, since a branch physically excludes some of the side-branches of smaller orders to have the same orientation. It is the exclusion by the radial main branches which causes the dips of $P(\phi, s)$ at $\phi=0$ in Fig. 3. This correlation also lead to the break down of any analogous power law decay of the amplitudes in the cosine expansion of $P(\phi, n)$. 


\section{DISCUSSIONS}

The local anisotropy of DLA is far from being stabilized for clusters of size $N \lesssim 10^{6}$. Assuming that Eq. (3) can be extrapolated to $N \rightarrow \infty$, we get $\lim _{N \rightarrow \infty} P(\theta, N)=1 / 2 \pi$ and DLA is locally isotropic asymptotically. The reliability of this extrapolation deserves special attention due to its simplicity, excellent agreement with simulation for $300 \lesssim N \lesssim 10^{6}$, and consistency with an intuitive phenomenological model. At finite $N$, the anisotropy can quantitatively be expressed by the proportion, $P_{F}$, of forward sticking events given by $P_{F} \simeq 0.5+2 a_{1} N^{-\gamma_{1}}$ from Eq. (四). For $N=10^{3}$ to $10^{6}, P_{F}$ decreases from $80 \%$ to $65 \%$. The anisotropy is still strong and agrees with the density correlation measurements «4. Achieving approximate isotropy with $P_{F} \lesssim 55 \%$ requires $N \gtrsim 10^{11}$ !

Our results on $P(\theta, N)$ is one of the most accurate non-trivial measurements ever done on DLA. In particular, the amazing accuracy in the one parameter fit to the simple analytic form in Eq. (1) at $N=614400$ is rare. The $\cos (\theta)$ term, which is the dominant term representing the anisotropy, was not identified before because forward and backward attachments are not properly discriminated in the correlation measurements [4].

We hope to motivate similar measurements for diffusion limited deposition (DLP). If analogous anisotropy tends to zero asymptotically, very large DLP might not be self-affine 12]. The same approach might also be attempted for the dielectric breakdown model [2].

The numerical part of this work was done with Henry Kaufman and Benoit B. Mandelbrot, who are gratefully acknowledged. 


\section{REFERENCES}

[1] T. A. Witten and L. M. Sander, Phys. Rev. Lett. 47, 1400 (1981)., Phys. Rev. B 27, 2586 (1983).

[2] T. Vicsek, Fractal growth phenomena, 2nd Ed. (World Scientific, Singapore 1992).

[3] H. Kaufman, unpublished.

[4] P. Meakin and T. Viscek, Phys. Rev. A 32, 685 (1985).

[5] B.B. Mandelbrot, The fractal geometry of nature (Freeman, San Francisco 1982).

[6] B.B. Mandelbrot and T. Viscek, J. Phys. A 22, L377 (1989).

[7] L.B. Leopold, American Scientist 50, 511 (1962).

[8] J. Feder, E.L. Hinrichsen, K.J. Måløy and T. Jøssang, Physica D 38, 104 (1989).

[9] P. Ossadnik, Phys. Rev. A 45, 1058 (1992).

[10] C.J.G. Evertsz and B.B. Mandelbrot, Physica A 177, 589 (1991).

[11] B.B. Mandelbrot, Physica A 19195 (191).

[12] P. Meakin, J. Kertész and T. Vicsek, J. Phys. A21 1271 (1988). 


\section{FIGURES}

FIG. 1. Probability distribution $P(\theta, N)$ of the angle of attachment $\theta$ for different cluster sizes $N$. Also shown are one parameter cosine fits for the data at $N=9600,76800$ and 614400 and two parameters fits for $N=150$ and 1200 .

FIG. 2. Amplitudes $a_{n}(N)$ of the cosine terms in the expansion of $P(\theta, N)$ as a function of cluster size $N$ : (a) $a_{1}$ to $a_{4}$ in semi-log plot; (b) $a_{1}$ and (c) $a_{2}$ in log-log plots respectively.

FIG. 3. Probability distribution $P(\phi, s)$ of the branch orientation angle $\phi$ for branches of order $s$ and fits with four cosine terms.

FIG. 4. Example of 200 particle probability flux lines with uniformly spaced starting position on a circle of radius $4 R_{G}$, where $R_{G}$ is radius of gyration of the one million particles DLA cluster. The potential was solved by over-relaxation method in a circular region of radius $4 R_{G}$ on a grid with lattice spacing $8 R_{G} / 7000$. Displayed region has radius $2 R_{G}$. 